\title{
Lidocaine-loaded non-ionic surfactant vesicles: characterization and in vitro permeation studies
}

\author{
M. Carafa ${ }^{\mathrm{a}, *}$, E. Santucci ${ }^{\mathrm{a}}, \mathrm{G}$. Lucania ${ }^{\mathrm{b}}$ \\ a Faculty of Pharmacy, University of Rome 'La Sapienza', P. le A: Moro 5, 00185 Rome, Italy \\ ${ }^{\mathrm{b}}$ Department of Experimental Medicine and Pathology, University of Rome 'La Sapienza', Rome, Italy
}

Received 28 February 2001; received in revised form 16 July 2001; accepted 19 July 2001

\begin{abstract}
Our research on topical application of lidocaine-loaded non-ionic surfactant vesicles (NSVs) was prompted by the great interest on new delivery systems for local anaesthetics. This study is focused on a novel formulation of NSVs entrapping lidocaine in the form of a free base (LID) and a hydrochloride (LIDHCl). NSVs were prepared from polyoxyethylene sorbitan monolaurate (Tween $20^{\mathrm{TM}}$ ) and cholesterol. The effect of vesicle composition and environmental $\mathrm{pH}$ condition (8.6-5.5) on drug encapsulation efficiency (e.e.) was investigated. Experimental strategies involved: freeze-fracture, microscopy technique, dynamic light scattering, permeation through Silastic ${ }^{\mathrm{TM}}$ and mouse abdominal skin, in vitro release kinetics of vesicle-entrapped drugs, fluorescence quenching analyses. Diffusion experiments showed that the flux of charged lidocaine through Silastic ${ }^{\mathrm{TM}}$ membrane was possible only after the vesicle encapsulation. Permeation through mouse abdominal skin of LIDHCl loaded vesicles showed a higher flux and a shorter lag time with respect to classical liposome formulations, while LID permeation rate was quite similar for NSV and liposome formulations. Vesicles were also prepared in the presence of dicetylphosphate (DCP) and $N$-cetylpyridinium chloride $(\mathrm{CP})$ to obtain negatively and positively charged vesicles respectively, but in this case the e.e. of the drug was negligible. The possible reason of the remarkable lower e.e. observed with charged vesicles was investigated by means of fluorescence quenching experiments. (c) 2002 Elsevier Science B.V. All rights reserved.
\end{abstract}

Keywords: Surfactant vesicles; Polysorbate 20; Lidocaine; Fluorescence quenching; Release rate; Permeation

\section{Introduction}

In the formulation of topical dosage forms, great attention has been devoted to new structures which can ensure either adequate localization of the drug within the skin to enhance the local

\footnotetext{
* Corresponding author. Tel.: + 39-649-913-775; fax: + 39649-913-133.

E-mail address: maria.carafa@uniroma1.it (M. Carafa).
}

effect or can increase the penetration through the stratum corneum and viable epidermis for a systemic effect.

For these purposes vesicular systems, liposomes in particular, have been investigated by several groups (Masini et al., 1993; Vutla et al., 1996).Vesicles, consisting of one or more surfactant bilayers enclosing aqueous spaces, have been considered of particular interest because they offer, over liposomes, several advantages with re- 
spect to chemical stability, lower costs and availability of materials.

Many attempts have been made to provide efficient anaesthesia of the skin or of mucosal tissues in case of pain itching and burning associated with cutaneous inflammatory response to different agents and with minor surgical operations. However, several studies indicated the limited efficiency on intact skin of available anaesthetic preparations (Dalili and Adriani, 1971) and the need of prolonged applications and high drug concentrations (Brechner et al., 1967; Lubens et al., 1974; Akerman, 1978; Ohlsen and Englesson, 1980).

The great interest in lidocaine delivery systems, as pointed out by the studies carried out in the last years (Stozek and Krowczynski, 1985; Gesztes and Mezei, 1988; Foldvari et al., 1990; Planas et al., 1992; Foldvari et al., 1993; Hou and Yu, 1997; Lener et al., 1997; Bucalo et al., 1998; Fisher et al., 1998; Karami and Beronius, 1998; Mircioiu et al., 1998; Görner et al., 1999; Malinovsky et al., 1999; Scherlund et al., 2000) prompted us to evaluate the possibility to load with lidocaine, as a free base and as hydrochloride, new vesicles obtained with polysorbate 20 and cholesterol (Santucci et al., 1996), already investigated for ophtalmic delivery (Saettone et al., 1996).

Aim of this investigation is to evaluate, by means of in vitro permeation experiments through a synthetic lipophilic barrier (Silastic ${ }^{\mathrm{TM}}$, Flynn and Rosenman, 1971) and through mouse abdominal skin, the behaviour of the new non-phospholipid vesicles in comparison with classical liposome and micellar formulations and to investigate the optimization of the drug encapsulation, taking into account that van Hal et al. (1996) reported, by in vitro studies, that the diffusion of lidocaine through human stratum corneum was not influenced by the encapsulation in non ionic surfactant vesicles.

The use of both a synthetic and a biological membrane allows to discriminate between the effects related to variations of thermodynamic parameters and those correlated to biological factors. Obtained results are explained in terms of entrapment and localization of the drug in the tested vesicle formulations. The lidocaine distribu- tion in the surfactant vesicles (NSVs) bilayers was studied by means of a new approach based on the evaluation of the fluorescence quenching dependent upon the interaction between the intramembrane fluorescent probe 12-(9-anthroyl)stearic acid (12-AS) and the anaesthetic (Koblin et al., 1975; Thulborn and Sawyer, 1978; Thulborn et al., 1979; Surewicz and Leyko, 1982). Ionic/non-ionic surfactant vesicles (i.e. charged NSVs) were also prepared (Carafa et al., 1998) and studied for the same purpose (to facilitate the vesicle-biological membrane interaction, Burnette and Bagnefski, 1988; Carafa et al., 1999), and some conclusions about their behaviour are drawn.

\section{Materials and methods}

\subsection{Materials}

Polysorbate 20 (TW20) and Cholesterol (CHOL) were purchased from Merck. Dicetylphosphate (DCP), $N$-pyridiniumcetylchloride (CP), Lidocaine (LID), Lidocaine hydrochloride (LIDHCl) and 12-(9-anthroyl)stearic acid (12-AS) were obtained from Sigma.

Phospholipon 90 was a generous gift of Nattermann Phospholipid. Sephadex G 75 was a Pharmacia product. Silastic nonreinforced, nonsterile membranes were provided by the Dow Corning and the nude mice CD2 -abdominal skin (mice 5-7 weeks old) were from Weizmann Institut, Israel.

All other products and reagents were of analytical grade. Distilled water was always used.

\subsection{Methods}

\subsubsection{Preparation of non-ionic surfactant vesicles}

The composition of the tested samples is reported in Table 1 and, as it can be observed, Tw20 concentration was always remarkably above CMC $\left(0.006 \mathrm{~g} / 100 \mathrm{ml}\right.$, in water, at $\left.20{ }^{\circ} \mathrm{C}\right)$.

Unilamellar vesicles were obtained by means of the 'film' method as previously reported (Carafa et al., 1999). The dried film was hydrated by addition of an aqueous phase (Phosphate buffer 
$\mathrm{pH} 8.6$ or 5.5). The dispersion was vortexed for $\sim 20 \mathrm{~min}$ and then sonicated, for $3 \mathrm{~min}$ at $60{ }^{\circ} \mathrm{C}$ (Vibracells-VCX400 (Sonics), equipped with an exponential microprobe operating at $23 \mathrm{KHz}$ and an amplitude of $6 \mathrm{~mm}$ ).

Due to the difference in solubility, in the case of vesicles prepared with lidocaine as a free base, the drug was added to the initial organic solvent while for the vesicles prepared with $\mathrm{LIDHCl}$ the drug was dissolved in the aqueous phase added to the surfactant/cholesterol dried film.

The overall LID and LIDHCl loading concentrations (i.e. free and entrapped in the vesicles) in the tested vesicle dispersions were $1,3,5,7 \% \mathrm{w} / \mathrm{v}$, respectively.

For the preparation of the fluorescent labelled vesicles, organic solutions $\left(\mathrm{CHCl}_{3} / \mathrm{CH}_{3} \mathrm{OH}-1 / 3\right)$ of the appropriate amounts of surfactant, cholesterol and 12-AS were mixed at a surfactant-probe molar ratio 100:1 and processed as described above to obtain the dry film. The film was hydrated by addition of the phosphate buffer ( $\mathrm{pH} 8.6$ or 5.5). Also in this case the dispersion was vortexed and then sonicated.

Conventional liposomes, used as reference, were prepared by means of the same technique carried out at room temperature and using appropriate amounts of Phospholipon 90 (Table 1). Phospholipid vesicles were prepared from $15 \mathrm{mM}$ lipid instead of $30 \mathrm{mM}$ surfactant/cholesterol mixture because these formulations gave the same drug encapsulation efficiency (Table 2).

\subsubsection{Vesicle purification}

In order to separate loaded NSVs from untrapped substances the vesicle dispersion was purified by gel-filtration on Sephadex G75 (glass column $50 \times 1.2 \mathrm{~cm}$ ), using phosphate buffer as eluent.

\subsubsection{Characterization of the vesicles}

2.2.3.1. Freeze-fracture. Vesicles were also examined by means of the freeze-fracture microscopy technique.

The samples were impregnated in $30 \%$ glycerol and then frozen into partially solidified Freon 22, freeze-fractured in a freeze-fracture device $\left(105^{\circ} \mathrm{C}, 10^{-6} \mathrm{mmHg}\right)$ and replicated by evaporation from a platinum/carbon gun.

The replicas were extensively washed with distilled water, picked up onto Formvar-coated grids and examined with a Philips CM 10 transmission electron microscope (Fig. 1).

\subsubsection{Size measurements and evaluation of vesicle} stability by dynamic light scattering (DLS).. The vesicle dispersions were diluted about 100 times in the same buffer, used for their preparation. Dust particles were eliminated by filtration $(0.45 \mu \mathrm{m})$.

Vesicles size distribution was measured on a Malvern Zetamaster ZEM 5002 (Malvern, UK) at $25^{\circ} \mathrm{C}$, with a scattering angle of $90.0^{\circ}$. The polidispersity index (p.i.) was then calculated (Table 3).

Table 1

Sample composition (mM)

\begin{tabular}{|c|c|c|c|c|c|c|c|}
\hline Sample & TW20 & CHOL & DCP & $\mathrm{CP}$ & $\mathrm{Ph} 90$ & LID & LIDHCl \\
\hline 1 & 15.0 & 15.0 & - & - & - & - & - \\
\hline $1 \mathrm{LIDHCl}$ & 15.0 & 15.0 & - & - & - & - & $2 \times 10^{2}$ \\
\hline 1LID & 15.0 & 15.0 & - & - & - & $2 \times 10^{2}$ & - \\
\hline 2 & 7.5 & 15.0 & 7.5 & - & - & - & - \\
\hline 3 & 14.0 & 15.0 & - & 1.0 & - & - & - \\
\hline 4LID & - & - & - & - & 15.0 & $2 \times 10^{2}$ & - \\
\hline
\end{tabular}

TW20, polyoxy-ethylene sorbitan monolaurate; CHOL, cholesterol; DCP, dicetylphosphate; CP, $N$-cetylpyridinium chloride; Ph 90, Phospholipon 90; LIDHCl, lidocaine hydrochloride; LID, lidocaine. In the table are reported only the drug-loaded formulations used for permeation experiments. 
Table 2

Drug encapsulation efficiency (e.e.) expressed as \% of loading concentration

\begin{tabular}{|c|c|c|c|c|c|c|c|c|}
\hline Sample & $\begin{array}{l}\text { LID } 1 \% ; \mathrm{pH} \\
5.5\end{array}$ & $\begin{array}{l}\text { LID 1\%; pH } \\
8.6\end{array}$ & $\begin{array}{l}\text { LIDHCl } 1 \% \text {; } \\
\text { pH } 5.5\end{array}$ & $\begin{array}{l}\text { LIDHCl 1\%; pH } \\
8.6\end{array}$ & $\begin{array}{l}\text { LID } 5 \% ; \mathrm{pH} \\
5.5\end{array}$ & $\begin{array}{l}\text { LID } 5 \% ; \mathrm{pH} \\
8.6\end{array}$ & $\begin{array}{l}\text { LIDHCl 5\%; pH } \\
5.5\end{array}$ & $\begin{array}{l}\text { LIDHCl 5\%; pH } \\
8.6\end{array}$ \\
\hline 1 & $2.0 \pm 0.1$ & $0.5 \pm 0.1$ & $1.1 \pm 0.1$ & $0.9 \pm 0.1$ & $30.0 \pm 0.1$ & $0.6 \pm 0.1$ & $32.0 \pm 0.1$ & $0.6 \pm 0.1$ \\
\hline 4 & $2.0 \pm 0.1$ & $0.7 \pm 0.1$ & $1.2 \pm 0.2$ & $0.9 \pm 0.2$ & $31.0 \pm 0.1$ & $0.5 \pm 0.1$ & $32.0 \pm 0.1$ & $0.6 \pm 0.1$ \\
\hline
\end{tabular}



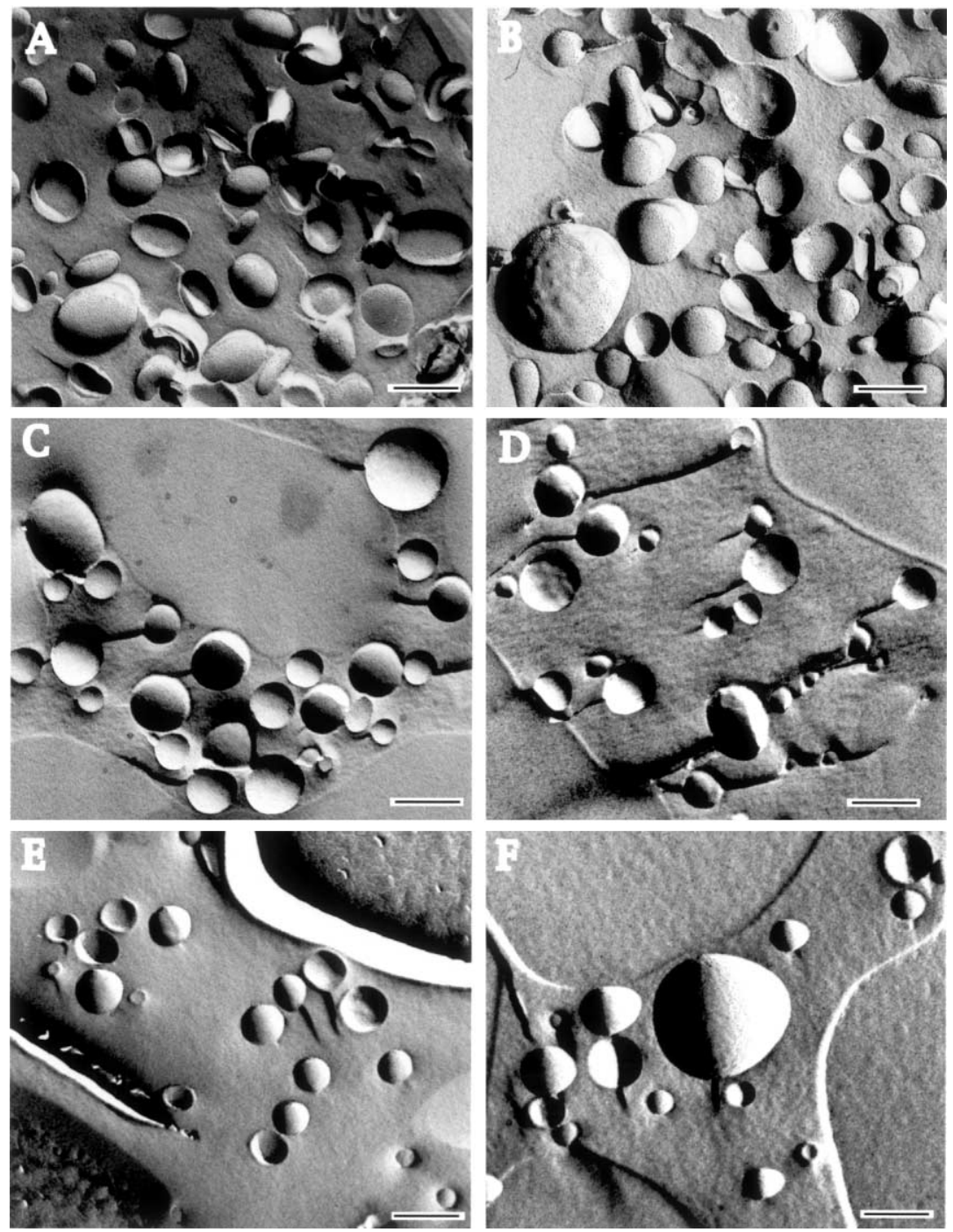

Fig. 1. Transmission electron micrographs of drug-loaded vesicles after freeze-fracture ; A, C and F: neutral, positively and negatively charged vesicles loaded with LIDHCl; B, D and F: neutral, positively and negatively charged vesicles loaded with LID. The bar corresponds to $0.2 \mathrm{~mm}$.

Vesicle stability, in terms of changes in vesicle dimensions after aggregation, was evaluated by means of the same technique on samples stored at 25 and $32{ }^{\circ} \mathrm{C}$.

\subsection{Drug encapsulation efficiency and in vitro release}

The drug entrapment within the vesicles was assessed by HPLC on purified vesicles, after their disruption with isopropanol (vesicle dispersion/ isopropanol 1/1). All analyses were carried out on a Perkin-Elmer 250 liquid chromatography apparatus, equipped with a Perkin-Elmer 235 photodiode array detector, a $20 \mu 1$ Rheodyne injector and a computer integrating apparatus.

The column was a Supelcogel ODP-50 (15 $\mathrm{cm} \times 4.00 \mathrm{~mm}$ I.D.); the mobile phase was a $40 / 60$ mixture of acetonitrile $/ 0.25 \mathrm{M}$ phosphate buffer ( $\mathrm{pH} 8.0$, adjusted with triethylamine). The use of 
a buffer of $\mathrm{pH}$ lower than 8.0 was not possible, because the affinity of the ionized form of lidocaine with stationary phase was negligible and the drug peak appear together with the solvent front. The flow rate was $0.25 \mathrm{ml} / \mathrm{min}$, the temperature was maintained at $30{ }^{\circ} \mathrm{C}$ and the detection was carried out at $255 \mathrm{~nm}$. Under the stated conditions, the retention time of both LID and LIDHCl was 10.2 minutes.

Drug encapsulation efficiency (e.e.) was calculated as follows (Görner et al., 1999):

$$
\text { e.e. }=100 \times \frac{\text { mass of incorporated drug }}{\text { mass used for vesicle preparation }}
$$

In vitro release experiments were performed in a flow-through apparatus (USP XXIV Ed) at $32{ }^{\circ} \mathrm{C}$ in a $\mathrm{pH} 5.5$ buffer. Fixed volumes of vesicle samples were included in dialysis sacs (cut-off $8.000)$ with a fixed diffusing area $\left(5.5 \mathrm{~cm}^{2}\right)$. Parallel measurements, carried out using $\mathrm{LIDHCl}$ without vesicles, proved that in no case the diffusion across the dialysis membrane was the limiting step of the overall diffusion process. The drug concentration was detected in the outer solution at fixed time intervals by means of the HPLC method described above, taking into account the dilution factor.

Also the surfactant concentration was determined in the outer solution and it remained under the CMC overall the release experiments. The permeability coefficient of the bilayer, $\mathrm{P}_{\mathrm{bl}}$, was determined using the linear expression (Ho et al., 1985)

$$
C_{\mathrm{b}}=C_{\mathrm{b}}(0)+\left(3 \mathrm{P}_{\mathrm{bl}} T / a(1-\varepsilon) V\right) t,
$$

where $C_{\mathrm{b}}$ and $C_{\mathrm{b}}(0)=$ solute concentrations in the bulk liquid at time $t$ and zero, respectively; $T=$ initial total amount of vesicle-entrapped solute

Table 3

Vesicle size $(\mathrm{nm})$

\begin{tabular}{lll}
\hline Sample & $\mathrm{nm}$ & p.i. \\
\hline 1LIDHCl & $170.2+11$ & 0.07 \\
1LID & $170.6+16$ & 0.06 \\
4LIDHCl & $167.7+16$ & 0.03 \\
4LID & $174.7+10$ & 0.03
\end{tabular}

(mg); $\varepsilon=$ volume fraction of vesicles, as determined by measurements after centrifugation; $V=$ volume of the suspension ( $\mathrm{ml}$ ) and $a=$ vesicle radius $(\mu \mathrm{m})$. The equation is valid for the period in which the amount of solute released is small.

\subsection{Fluorescence quenching analyses}

In order to study the interaction of lidocaine with vesicle bilayers, labelled with 12-AS, small amounts $(50 \mu \mathrm{l})$ of a concentrated solution $(20 \%$ $\mathrm{w} / \mathrm{v}$ ) of the local anaesthetic were added to $3 \mathrm{ml}$ of a vesicle dispersion and magnetically stirred for $5 \mathrm{~min}$. The dispersion was then left to equilibrate for $10 \mathrm{~min}$ before fluorescence measurements.

A Perkin-Elmer LS 5 Spectrofluorometer was used and emission scanning (400-560 nm; excitation $386 \mathrm{~nm}$ ) was recorded on PE Data Station. Reference unlabelled vesicles and lidocaine, at the tested concentrations, did not exhibit any detectable fluorescence.

Turbidity measurements $\left(E_{\mathrm{x}} / E_{\mathrm{m}} 600 / 600 \mathrm{~nm}\right)$ were performed before drug addition and at the end of fluorescence analyses to confirm that no detectable vesicle disruption occurred.

The influence of quencher concentration on fluorescence intensity was measured using the equation:

$F_{\mathrm{o}} / F_{\mathrm{t}}=1+K q(Q)$,

where $F_{\mathrm{o}}$ and $F_{\mathrm{t}}$ are the fluorescence intensities determined before and after the various additions of the quencher; $(Q)$ is the quencher concentration and $K q$ is the quenching constant (Surewicz and Leyko, 1982). Thus a plot of $F_{\mathrm{o}} / F_{\mathrm{t}}$ versus $(Q)$ gives a straight line of slope $K q$ and an intercept of one.

Since quenching depends upon the molecular contact between quencher and fluorophore, measures of fluorescence quenching with 12-AS, deeply located within the membranes, can be used to localize the quencher (Lakowicz, 1986).

\subsection{Diffusion experiments}

Diffusion experiments were carried out in sideby-side diffusion cells (Valia Chien) for $24 \mathrm{~h}$, at $32{ }^{\circ} \mathrm{C}$, through the Silastic ${ }^{\mathrm{TM}}$ membrane or the 
abdominal skin of male nude mice, under continuous stirring, using non purified vesicle preparations (drug concentration 5\% w/v, partially entrapped in the vesicular structures).

The exact half cell volumes $(3.2 \mathrm{ml})$ and surface areas $\left(0.64 \mathrm{~cm}^{2}\right)$ have been taken into account when analyzing permeation data.

Even if no solubility problems are expected at $\mathrm{pH} 7.4$ (the drug is $>50 \%$ charged), the receiver content was $1 / 1$ ethanol/ $\mathrm{pH} 7.4$ buffer, in order to ensure pseudo-sink conditions.

Before each experiment the system was allowed to equilibrate for $1 \mathrm{~h}$. At fixed time intervals, $50 \mu \mathrm{l}$ samples were drawn from the acceptor compartment and the lidocaine concentration was determined by HPLC, as described above and an equivalent amount of ethanol/buffer solution was added each time to maintain a constant volume in the receiver compartment. Dilution of the receiver medium was taken into account when processing the permeation data.

The diffusion rate of vesicular systems through Silastic $^{\mathrm{TM}}$ and skin were compared to drug/Tween 20 dispersions with the same concentration for the drug.

At the end of each experiment the skin was removed, cleaned and extracted with a $50 \% \mathrm{w} / \mathrm{w}$ hydroethanolic solution.

The cumulative amount of LID or LIDHCl that permeated through the membrane/skin after the $n$th sampling (Qn) was estimated by Eq. (3) (Yu and Liao, 1966)

$$
Q n=C_{n} V+\sum_{i=1}^{n-1} V_{\mathrm{s}} C_{i},
$$

where $C_{i}$ and $C_{n}$ are the various measured concentrations from 1 to $n, V$ is the volume of the solution in the receptor compartment and $V_{\mathrm{s}}$ is the sampling volume. Other skin permeation kinetic parameters (flux, permeation constant and lag time) were calculated using the Transderm Software for data analysis, elaborated by Touitou and Watenfeld (1987).

Each system was tested in two different cells and the experiments were repeated three times. The obtained data were compared using the Mann-Whitney test, the Krustall Wallis test (non parametric ANOVA) and the Dunnis multiple comparison test. In all cases the $P$ values are significant $(P<0.05)$.

\section{Results and discussion}

We characterized three different surfactant vesicle formulations (Table 1) evaluating the entrapment efficiency (Table 2) at two different $\mathrm{pH}$ values (i.e. 5.5 and 8.6). Phosphatidilcoline liposomes were used as reference.

In a set of experiments the aqueous phase was buffered at $\mathrm{pH} 5.5$, similar to that of the skin, which assured that most Lidocaine was positively charged, another group of experiments was carried out at $\mathrm{pH} 8.6$ to maintain most Lidocaine in its uncharged form $\left(\mathrm{p} K_{\mathrm{a}}=7.86\right)$. According to Watts and Poile (1986) it can in fact be assumed that the $\mathrm{p} K_{\mathrm{a}}$ of the local anaesthetic is, at the vesicle surface, identical to its value in solution.

Furthermore, it must be pointed out that lidocaine has a melting point at $68{ }^{\circ} \mathrm{C}$ thus sonication at $60{ }^{\circ} \mathrm{C}$ does not result in the formation of the melted phase of the lidocaine base, as reported by van Hal et al. (1996), who stated that niosomes cannot be formed with lidocaine above its melting point. Consequently it was possible to prepare NSVs using lidocaine also in the form of a free base; while in most studies and in the marketed formulations this local anaesthetic is used in the hydrochloride form.

At $\mathrm{pH} 8.6$ the amount of lidocaine entrapped in NSVs was negligible for all tested formulations (Table 2); at $\mathrm{pH} 5.5$ the best entrapment efficiency was obtained for sample 1 prepared with $5 \% \mathrm{w} / \mathrm{v}$ lidocaine; for lower anaesthetic concentrations the e.e. was lower while for $7 \% \mathrm{w} / \mathrm{v}$ formulations there was no vesicle formation at all. For the charged vesicles remarkably low e.e. are obtained at all tested initial LID and $\mathrm{LIDHCl}$ concentrations $(1-7 \% \mathrm{w} / \mathrm{v})$, even at $\mathrm{pH} 5.5$ (Table 2).

Considering e.e. values, in spite of our interest in charged NSV formulations, we focused our investigation, at least as far as diffusion and release studies are concerned, on the neutral sample prepared with $5 \% \mathrm{w} / \mathrm{v}$ loading concentration at pH 5.5. 


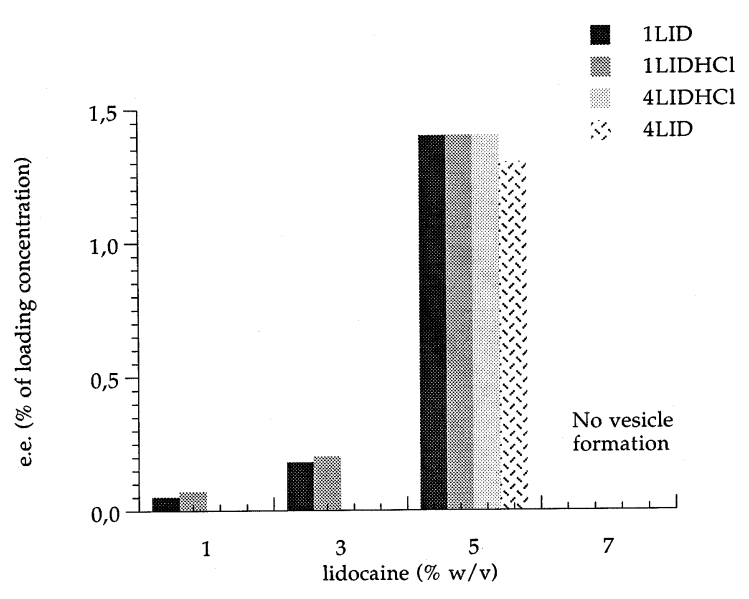

Fig. 2. Influence of the loading concentration on the encapsulation efficiency of sample 1 (the e.e. at $5 \% \mathrm{w} / \mathrm{v}$ lidocaine loading concentration of sample 4 , used as reference, is also reported).

In order to evaluate the specific effect of the encapsulation in non-phospholipid vesicles on lidocaine permeation through biological and synthetic barriers, we compared the diffusion through mouse skin and through Silastic ${ }^{\mathrm{TM}}$ of LID and LIDHCl from NSVs and from classical liposome preparation.

It is known (Cappel and Kreuter, 1991) that aqueous solutions of polysorbate 20 do not modify the permeability of methanol through Silastic $^{\mathrm{TM}}$ and mouse skin significantly, while the permeability of octanol decreases as a function of polysorbate concentration. This last effect was explained as a result of the decrease in thermodynamic activity due to micellar complexation of the lipophilic octanol.In our experiments the permeation of LIDHCl through the Silastic ${ }^{\mathrm{TM}}$ lipophilic membrane was not detectable in absence of Tween 20.

It was evidenced that permeation rates of dispersions of LID and LIDHCl in presence of the surfactant, not in vesicular structure but as micelles, are quite low but detectable and different (i.e. for LID $1.54 \times 10^{-2} \mathrm{ng} / \mathrm{cm}^{2} / \mathrm{h}$ and for LID$\mathrm{HCl} 2.5 \times 10^{-3} \mathrm{ng} / \mathrm{cm}^{2} / \mathrm{h}$ ) even if at $\mathrm{pH} 5.5$ most of lidocaine is positively charged (Fig. 3(a)). We can assume that the higher diffusion of LID with respect to $\mathrm{LIDHCl}$ can be related to the presence of the free base, solubilized within the core of surfactant micelles, but anyhow capable to diffuse through the lipophilic barrier, because of the rapid equilibrium between monomers and micelles. The different diffusion rates of LID and LIDHCl observed in the presence of micelles is even more remarkable when the drug, in its different forms, is entrapped within liposomes and NSVs (Fig. 3(b)). In this specific case it must be again pointed out that Silastic ${ }^{\mathrm{TM}}$ represents a totally inert lipophilic barrier and this can explain why the diffusion from 'classical' liposomes is higher than that detected with NSVs, that are in all cases more hydrophilic.

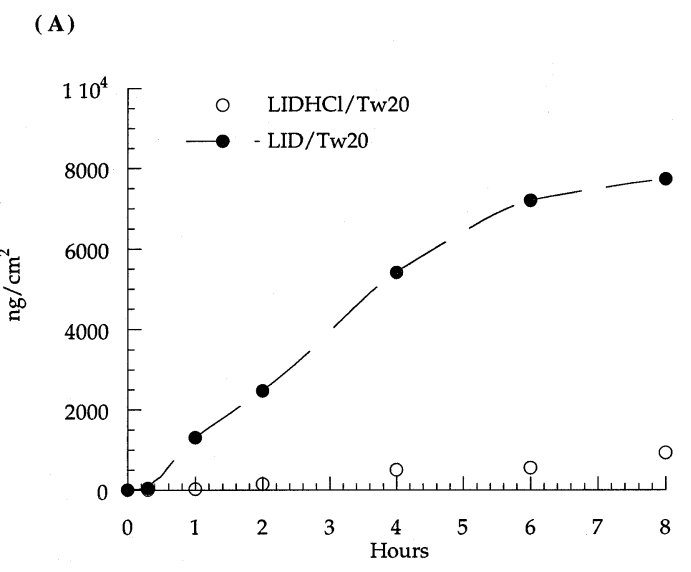

(B)

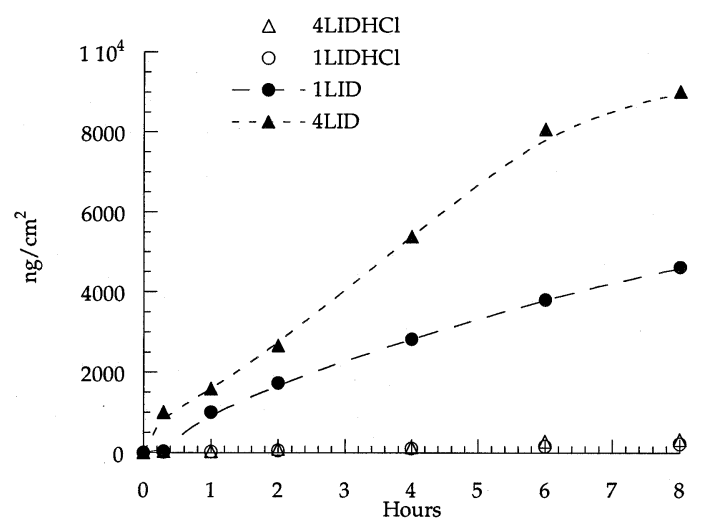

Fig. 3. (A) Permeation of LIDHCl and LID in presence of Tween 20, at the same concentration used for SV formulations (above CMC) compared to (B) permeation of $\mathrm{LIDHCl}$ and LID-loaded sample 1 and sample 4 through Silastic ${ }^{\mathrm{TM}}$, at $32{ }^{\circ} \mathrm{C}$. 
Table 4

Drug permeation through mouse skin.

\begin{tabular}{lllll}
\hline Sample & Flux $(\mathrm{mg} / \mathrm{h} / \mathrm{cm})$ & $K_{\mathrm{p}}{ }^{\mathrm{b}}(\mathrm{cm} / \mathrm{h})$ & $Q_{\text {cum }}{ }^{\mathrm{a}}(\mathrm{mg})$ & Lag time \\
\hline 1LIDHCl & $6.6 \times 10^{-3}$ & $1.30 \times 10^{-4}$ & $0.12+0.09$ & $1 \mathrm{~h} 25 \mathrm{~min}$ \\
1LID & $1.28 \times 10^{-2}$ & $2.58 \times 10^{-4}$ & $0.33+0.10$ & $1 \mathrm{~h}$ \\
4LIDHCl & $2.44 \times 10^{-3}$ & $4.79 \times 10^{-4}$ & $0.05+0.02$ & $2 \mathrm{~h} 10 \mathrm{~min}$ \\
4LID & $1.27 \times 10^{-2}$ & $2.60 \times 10^{-4}$ & $0.32+0.13$ & $1 \mathrm{~h} 16 \mathrm{~min}$ \\
\hline
\end{tabular}

${ }^{\text {a }} Q_{\text {cum }}$, cumulative amounts of permeated formula $( \pm \mathrm{SD})$ after $8 \mathrm{~h}$.

${ }^{\mathrm{b}} K_{\mathrm{p}}$, permeation constant.

On the other hand, in skin permeation experiments, the obtained results (Table 4, Fig. 4) indicate that encapsulation in NSVs of LID and LIDHCl leads in all cases to a faster diffusion with respect to the corresponding classical liposome formulations. This effect can probably be related to the enhancing effect of the hydrophilic surfactant Tw20 (van Boxtel et al., 1990; Arellano et al., 1998), not present with a totally lipophilic membrane like Silastic ${ }^{\mathrm{TM}}$. Furthermore, as expected, for LIDHCl-loaded vesicles the skin permeation kinetics parameters are lower than those obtained with LID (Table 4).

Reported diffusion results, observed with Silastic $^{\mathrm{TM}}$ or mouse skin, cannot be related to different release kinetic, from Eq. (1), from vesicular structures because release studies from the dialysis sacs showed that for NSVs (i.e. sample 1) and liposomes (i.e. sample 4) there were no detectable differences for LID and LIDHCl loaded formulations (Table 5).

Furthermore, the differences in permeation experiments cannot be related to vesicle dimensions and e.e. values, because they were quite similar for the different tested formulations (Table 2, Fig. 2 ), or to different stability of the vesicular structures because stability tests, carried out by Dynamic Light Scattering, on samples 1 and 4 showed that at 25 and $32{ }^{\circ} \mathrm{C}$ no significant variations of vesicle dimensions occur for at least 3 weeks.

In the light of the unresolved controversial issue of the mechanism of transdermal penetration off/ from vesicular carriers further experiments on vesicle/skin interaction, carried out by means of micoscopy analyses (freeze-fracture, SEM, confocal microscopy) are in progress.
As pointed out above, e.e. of both LID and LIDHCl in the charged vesicles was almost negligible, thus in vitro diffusion experiments were carried out only on neutral vesicle formulations. Nevertheless it appeared interesting to investigate the reason of such remarkable different behaviour.

In order to explain the differences of e.e. between neutral and charged vesicles, fluorescence quenching analyses were carried out. Addition of LIDHCl to 12-AS solutions decreased fluorescence intensity without significant changes in the shape of the emission spectrum. The observed decrease in emission intensity showed that LID$\mathrm{HCl}$ may actually quench 12-AS fluorescence.

Fluorescence quenching experiments carried out with sample 1, prepared at $\mathrm{pH} 5.5$, gave a linear plot according to Eq. (2) when $F_{\mathrm{o}} / F_{\mathrm{t}}$ was reported versus $\mathrm{LIDHCl}$ concentrations (Fig. 5),

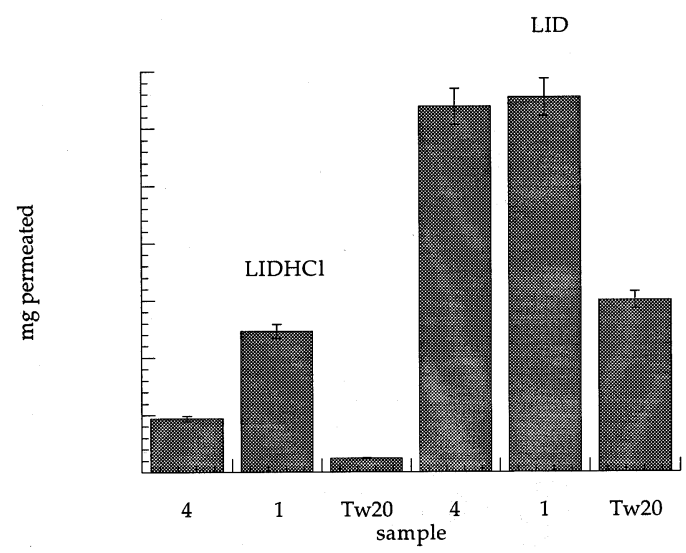

Fig. 4. Permeated amount of LID and LIDHCl through mouse skin, at $32^{\circ}$; Tween 20 concentration is the same used for vesicle formulations (above $\mathrm{CMC}$ ). 
Table 5

Permeability coefficients of the bilayers $( \pm \mathrm{SD})$ after $8 \mathrm{~h}$

\begin{tabular}{ll}
\hline Sample & Permeability coefficient $(\mathrm{cm} / \mathrm{h})$ \\
\hline 1LID & $2.83 \times 10^{-5}+0.05$ \\
1LIDHCl & $2.79 \times 10^{-5}+0.07$ \\
4LID & $2.81 \times 10^{-5}+0.05$ \\
4LIDHCl & $2.80 \times{ }^{-5}+0.04$ \\
\hline
\end{tabular}

because of the interaction of 12-AS with lidocaine. Since the fluorescent lipophilic probe is entrapped within the vesicle bilayers, the observed fluorescence quenching clearly indicates that lidocaine is located also in the bilayer.

This result is in agreement with the data reported by Mueller-Goymann and Frank (1986) who showed, by means of X-ray diffraction, that both lidocaine and lidocaine- $\mathrm{HCl}$ interacted with lamellar bilayers, composed of neutral surfactant (soyasterol-PEG-ether), and it is in agreement with the lidocaine molecule interaction with the hydrophobic moiety of Tweens reported by Goddart and Anathapadmanabhan (1993). The quenching constant $K q$ is dependent upon quencher (i.e. lidocaine) partition between vesicle membrane and the aqueous phase and upon diffusion rate from water into the more lipophilic bilayer (Lakowicz, 1986). The linear plots, related to the interaction of 12-AS with lidocaine in the vesicle bilayer, present different slope values for the various formulations.

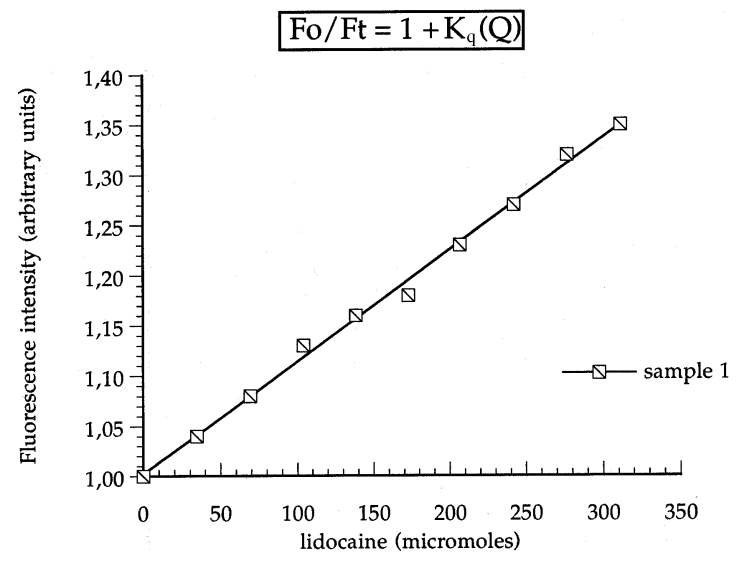

Fig. 5. Fluorescence intensity variation for AS-loaded neutral vesicles as a function of lidocaine amount (micromoles).
The quenching constant $K q$, calculated for charged vesicles (sample 2 and 3) is lower than that obtained with neutral NSVs (sample 1) thus it can be assumed that the presence of a net charge in the vesicles reduced lidocaine diffusion as observed also by Webb et al. (1995) with cationic liposomes and by van Hal et al. (1996) with negatively charged membrane.

Furthermore, the remarkable reduction of lidocaine encapsulation observed for charged vesicles (samples 2 and 3) can be explained by a lower partition of the drug in the vesicle bilayer with respect to neutral vesicles, even if it is not possible to distinguish between lidocaine entrapped in the water phase and lidocaine intercalated in the bilayers.

\section{Conclusions}

Reported results indicate that it is possible to load Tween 20/cholesterol vesicles with lidocaine and lidocaine hydrochloride.

When inserted in the vesicles, LID and LIDHCl are capable to diffuse through a model lipophilic membrane (Silastic ${ }^{\mathrm{TM}}$ ) and through mouse skin. In particular the performance of NSVs containing $\mathrm{LIDHCl}$ is remarkably better than that observed with classical liposomes and Tween 20 micelles.

Furthermore the obtained data provide direct evidence that neutral vesicles, prepared with Tween 20 and cholesterol, entrap a higher lidocaine amount, at $\mathrm{pH} 5.5$, than positively and negatively charged vesicles, as confirmed by fluorescence quenching analyses.

\section{Acknowledgements}

The authors are grateful to Professor Elka Touitou and her research group at the School of Pharmacy of The Hebrew University of Jerusalem (Israel) for their fundamental support in dynamic light scattering and ex vivo permeation experiments, as well as for stimulating suggestions and discussion. 


\section{References}

Akerman, B., 1978. Percutaneous local anaesthesia. Problems-solutions. Acta Anaesth. Scand. 70, 90-91.

Arellano, A., Santoyo, S., Martn, C., Ygartua, P., 1998. Surfactant effects on the in vitro percutaneous absorption of diclofenac sodium. Eur. J. Drug Metab. Pharmacokinet. 23, 307-312.

Brechner, V.L., Cohen, D.D., Pretsky, I., 1967. Dermal anaesthesia by the topical application of tetracaine base dissolved in dimethylsulfoxide. Ann. NY Acad. Sci. 141, 524-531.

Bucalo, B.D., Mirikitani, E.J., Moy, R.L., 1998. Comparison of skin anaesthetic effect of liposomal lidocaine, non liposomal lidocaine and EMLA, using 30-minute application time. Dermatol. Surg. 24, 537-541.

Burnette, R.R., Bagnefski, T.M., 1988. Influence of constant current iontophoresis on the impedance and passive $\mathrm{Na}^{+}$ permeability of excised nude mouse skin. J. Pharm. Sci. 77, 492-497.

Cappel, M.J., Kreuter, J., 1991. Effect of nonionic surfactants on trasdermal drug delivery: I. Polysorbates. Int. J. Pharm. 69, $143-153$.

Carafa, M., Santucci, E., Alhaique, F., Coviello, T., Murtas, E., Riccieri, F.M., Lucania, G., Torrisi, M.R., 1998. Preparation and properties of new unilamellar non-ionic/ionic surfactant vesicles. Int. J. Pharm. 160, 51-59.

Carafa, M., Lucania, G., Marchei, E., Dolo, V., Gianmatteo, M., Torrisi, M.R., Santucci, E., Pavan, A., 1999. Interaction of charged surfactant vesicles (SVs) with cultured human cells: a microscopy investigation". Biotech. Histochem. 743, 1-8.

Dalili, H., Adriani, J., 1971. The efficacy of local anesthetics in blocking the sensations of itch, burning and pain in normal and 'sunburned' skin. Clin. Pharmacol. Ther. 12, 913-919.

Fisher, R., Hung, O., Mezei, M., Stewart, R., 1998. Topical anaesthesia of intact skin: liposome-encapsulated tetracaine vs. EMLA. Br. J. Anaesth. 81, 972-973.

Flynn, G.L., Rosenman, T.J., 1971. Membrane diffusion II: influence of physical adsorption on molecular flux through heterogeneous dimethylpolysiloxane barriers. J. Pharm. Sci. 60, 1788-1796.

Foldvari, M., Gesztes, A., Mezei, M., 1990. Dermal drug delivery by liposome encapsulation: clinical and electron microscopy studies. J. Microencapsul. 7, 479-489.

Foldvari, M., Jarvis, B., Oguejifor, C.J.N., 1993. Topical dosage form of liposomal tetracaine: effect of additives on in vitro release and in vivo efficacy. J. Control. Rel. 27, 193-205.

Gesztes, A., Mezei, M., 1988. Topical anesthesia of the skin by liposome-encapsulated tetracaine. Anesth. Analg. 67, 1079-1081.

Goddart, E.D., Anathapadmanabhan, G., 1993. Interaction of surfactants with polymers and proteins, CRC Press, Boca Raton, FL, pp. 345-348

Görner, T., Gref, R., Michenot, D., Sommer, F., Tran, M.N., Dellacherie, E., 1999. Lidocaine-loaded biodegradable nanospheres. I. Optimization of the drug incorporation into the polymeric matrix. J. Control. Rel. 57, 259-268.

Ho, N.F.H., Ganesan, M.G., Weiner, N.D., Flynn, G.L., 1985. Mechanism of topical delivery of liposomally entrapped drugs. J. Control. Rel. 2, 61-65.

Hou, S.M., Yu, H.Y., 1997. Comparison of systemic absorption of aqueous and liposomal lidocaine following intra-articular injection in rabbit. J. Formos. Med. Assoc. 96, $141-143$.

Karami, K., Beronius, P., 1998. On iontophoretic delivery enhancement ionization of lidocaine hydrochloride in propylene glycol. Proceed. Int. Symp. Control. Rel. Bioact. 25, 615-616.

Koblin, D.D., Pace, W.D., Wang, H.H., 1975. The penetration of local anaesthetics into the red blood cell membrane as studied by fluorescence quenching. Arch. Bioch. Biophys. $171,176-182$.

Lakowicz, J.R., 1986. Principles of Fluorescence Spectroscopy, Plenum Press, New York, pp. 257-295.

Lener, E.V., Bucalo, B.D., Kist, D.A., Moy, R.L., 1997. Topical anaesthetic agents in dermatological surgery. A review. Dermatol. Surg. 23, 673-683.

Lubens, H.M., Ausdenmoore, R.W., Shafer, A.D., Reece, R.M., 1974. Anesthetic patch for painful procedures such as minor operations. Am. J. Dis. Child. 128, 192-194.

Malinovsky, J.M., Le Corre, P., Meunier, P., Chevanne, P., Pinaud, M., Leverge, R., Legros, F., 1999. A dose-response study of epidural liposomal bupivacaine in rabbits. J. Control. Rel. 28, 111-119.

Masini, V., Bonte, F., Meybeck, A., Wepierre, J., 1993. Cutaneous bioavailability in hairless rats of Tretinoin in liposomes gel. J. Pharm. Sci. 82, 17-24.

Mircioiu, C., Georgescu, C., Keller, O., Olteanu, M., 1998. Study of the rheological behaviour of a Carbopol gel containing lidocaine and Tween 40. Proceedings of the Second World Meeting APGI/APV

Mueller-Goymann, C.C., Frank, S.G., 1986. Interaction of lidocaine and lidocaine- $\mathrm{HCl}$ with the liquid crystal structure of topical preparations. Int. J. Pharm. 29, 147-159.

Ohlsen, L., Englesson, S., 1980. New anaesthetic formulation for epicutaneous application tested for cutting split skin grafts. Br. J. Anaest. 52, 413-417.

Planas, M.E., Gonzales, P., Rodriguez, L., Sanchez, S., Cevc, G., 1992. Noninvasive percutaneous induction of topical analgesia by new type of drug carrier and prolongation of local pain insensitivity by anaesthetic liposomes. Anesth. Analg. 75, 615-621.

Saettone, M.F., Perini, G., Carafa, M., Santucci, E., Alhaique, F., 1996. Non-ionic surfactant vesicle as ophtalmic carriers for cyclopentolate. A preliminary evaluation. STP Pharma Sci. 6, 94-98.

Santucci, E., Carafa, M., Coviello, S., Murtas, E., Riccieri, F.M., Alhaique, F., Modesti, A., Modica, A., 1996. Vesicle from polysorbate 20 and cholesterol. A simple preparation and a characterization. STP Pharma Sci. 6, 29-32.

Scherlund, M., Malmsten, M., Holmqvist, P., Brodin, A., 2000. Thermosetting microemulsions and mixed micellar 
solutions as drug delivery system for periodontal anesthesia. Int. J. Pharm. 194, 103-116.

Stozek, T., Krowczynski, L., 1985. Zum Einfluss des Einschlusses von Procainhydrochlorid in Liposomen auf die lokalanasthetische Wirkung. Pharmazie 44, 466468.

Surewicz, W.K., Leyko, W., 1982. Interaction of local anaesthetics with model phospholipid membranes. The effect of $\mathrm{pH}$ and phospholipid composition studied by quenching of an intramembrane fluorescent probe. J. Pharm. Pharmacol. 34, 359-363.

Thulborn, K.R., Sawyer, W.H., 1978. A microviscosity barrier in the lipid bilayer due to the presence of phospholipids containing unsaturated acyl chains. Biochim. Biophys. Acta $511,125-140$.

Thulborn, K.R., Tilley, L.M., Sawyer, W.H., Treolar, F.E., 1979. The use of $n$-(9-antranoyloxy)fatty acids to determine fluidity and polarity gradients in phospholipids bilayers. Biochim. Biophys. Acta 558, 166-178.

Touitou, E., Watenfeld, R., 1987. Transderm. Life Sci. 1

van Boxtel, R.M., Lambrecht, R.S., Collins, M.T., 1990. Effect of polyoxyethylene sorbate compounds (Tweens) on colonial morphology, growth, and ultrastructure of $M y$ cobacterium paratuberculosis. APMIS 98, 901-908.

van Hal, D.A., Jeremiasse, E., de Vringer, T., Junginger, H.E., Bouwstra, J.A., 1996. Encapsulation of lidocaine base and hydrochloride into non-ionic surfactant vesicles (NSVs) and diffusion throughout human stratum corneum in vitro. Eur. J. Pharm. Sci. 4, 147-157.

Vutla, N.B., Betageri, G.V., Banga, A.K., 1996. Transdermal iontophoretic delivery of Enkephalin formulated in liposomes. J. Pharm. Sci. 85, 5-10.

Watts, A., Poile, T.W., 1986. Direct determination by deuterium NMR of the ionization state of phospholipid and of local anaesthetic at the membrane surface. Biochim. Biophys. Acta 861, 362-372.

Webb, M.S., Wheeler, J.J., Bally, M.B., Mayer, L.D., 1995. The cationic lipid stearylamine reduces the permeability of cationic drug verapamil and prochloperazine to lipid bilayers: implications for drug delivery. Biochim. Biophys. Acta 1238, 147-155.

Yu, H., Liao, H., 1966. Triamcinolone permeation from different liposome formulations through rat skin in vitro. Int. J. Pharm. 127, 1-7. 